\title{
Long-time behavior of a Hele-Shaw type problem in random media
}

\author{
NORBERT POŽÁR \\ Department of Mathematics, UCLA, \\ Box 951555, Los Angeles, CA 90095-1555, USA \\ E-mail: npozar@math.ucla.edu
}

[Received 20 June 2010 and in revised form 15 February 2011]

\begin{abstract}
We study the long-time behavior of an exterior Hele-Shaw problem in random media with a free boundary velocity that depends on the position in dimensions $n \geqslant 2$. A natural rescaling of solutions that is compatible with the evolution of the free boundary leads to the homogenization of the free boundary velocity. By studying a limit obstacle problem for a Hele-Shaw problem with a point source, we are able to show the uniform convergence of the rescaled solution to a self-similar limit profile and we deduce that the rescaled free boundary uniformly approaches a sphere.
\end{abstract}

\section{Introduction}

We consider a Hele-Shaw type problem (HS) in random media and study the long-time behavior of the solutions. Let $n \geqslant 2$ and let $K \subset \Omega_{0} \subset \mathbb{R}^{n}, 0 \in$ int $K, K$ be a nonempty compact set and $\Omega_{0}$ be a bounded open set such that $K$ and $\Omega_{0}$ have smooth boundaries (see Figure 1). Let $\Gamma=\partial K$ and $\Gamma_{0}=\partial \Omega_{0}$. Formally, the Hele-Shaw type problem is to find a function $v(x, t): \mathbb{R}^{n} \times[0, \infty) \rightarrow \mathbb{R}$ that solves

$$
\begin{cases}-\Delta v=0 & \text { in }\{v>0\} \backslash K, \\ v=1 & \text { on } K, \\ v_{t}=g(x, \omega)|D v|^{2} & \text { on } \partial\{v>0\}, \\ v(\cdot, 0)=v_{0} & \text { on } \Omega_{0} \backslash K,\end{cases}
$$

where the initial data $v_{0}(x): \mathbb{R}^{n} \rightarrow \mathbb{R}$ is the solution of

$$
\begin{cases}-\Delta v_{0}=0 & \text { in } \Omega_{0} \backslash K, \\ v_{0}=1 & \text { on } K, \\ v_{0}=0 & \text { on } \partial \Omega_{0} .\end{cases}
$$

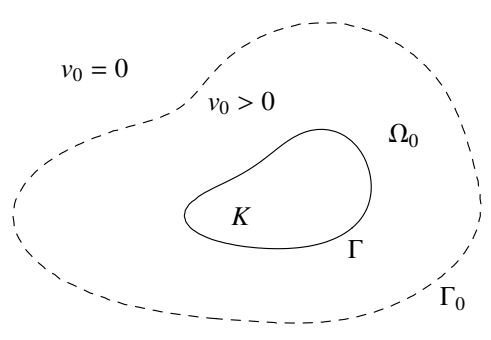

FIG. 1. Initial configuration. 
Here $D v$ and $\Delta v$ are, respectively, the gradient and the Laplacian of $v$ with respect to the space variable $x$, and $v_{t}$ is the partial derivative of $v$ with respect to the time variable $t$.

Let $(A, \mathcal{F}, \mu)$ be a probability space. We consider $g(x, \omega): \mathbb{R}^{n} \times A \rightarrow \mathbb{R}$, a continuous function in $x$ for a.e. $\omega \in A$, satisfying

$$
0<m \leqslant g(x, \omega) \leqslant M \quad \text { for all } x \in \mathbb{R}^{n} \text { and a.e. } \omega \in A,
$$

where $m$ and $M$ are positive constants. Furthermore, in order to observe some averaging behavior as $t \rightarrow \infty$, we assume that $g$ is stationary ergodic. In other words, we assume that we have a group $\left\{\tau_{x}\right\}_{x \in \mathbb{R}^{n}}$ of measure preserving transformations $\tau_{x}: A \rightarrow A$ such that

$$
g\left(x+x^{\prime}, \omega\right)=g\left(x^{\prime}, \tau_{x} \omega\right) \quad \text { for all } x, x^{\prime} \in \mathbb{R}^{n} \text { and a.e. } \omega \in A,
$$

i.e. $g$ is stationary. Furthermore, we require that $\left\{\tau_{x}\right\}_{x \in \mathbb{R}^{n}}$ is ergodic, that is, if $B \subset A$ is such that $\tau_{x}(B)=B$ for all $x \in \mathbb{R}^{n}$, then $\mu(B)=0$ or 1 . For a more detailed discussion of stationary ergodic media, see for instance [5,20].

The classical Hele-Shaw problem in two dimensions with $g \equiv 1$ was introduced in [12] modelling a slow movement of a viscous fluid injected in between two parallel plates close to each other that form the so-called Hele-Shaw cell. This problem naturally generalizes to all dimensions $n \geqslant 1$.

We study a Hele-Shaw type problem (HS) describing a pressure-driven motion of a fluid in an inhomogeneous random medium where the velocity law of the fluid on the free boundary depends on the position. The homogenization of this problem was recently studied in [14]. Free boundary problems with similar velocity laws have various applications in the plastics industry [22, 24, 28], in electromechanical machining [19] and others. In fact, Hele-Shaw problem can be thought of as a quasi-stationary limit of the Stefan problem with a similar boundary velocity law, modelling heat transfer [2, 17, 21,27].

In this paper, we are concerned with the behavior of the solution for large times. The main result, Theorem 7.1 , is the locally uniform convergence of a rescaled viscosity solution of the inhomogeneous problem (HS),

$$
v^{\lambda}(x, t)=\lambda^{(n-2) / n} v\left(\lambda^{1 / n} x, \lambda t\right) \quad \text { if } n \geqslant 3
$$

(see Section 3 for a discussion of the rescaling and the case $n=2$ ), which then satisfies

$$
v_{t}^{\lambda}=g\left(\lambda^{1 / n} x, \omega\right)\left|D v^{\lambda}\right|^{2} \quad \text { on } \partial\left\{v^{\lambda}>0\right\},
$$

and its free boundary as $\lambda \rightarrow \infty$ to the self-similar solution of the Hele-Shaw problem with a point source, formally

$$
\begin{cases}-\Delta v=C \delta & \text { in }\{v>0\}, \\ v_{t}=\frac{1}{\langle 1 / g\rangle}|D v|^{2} & \text { on } \partial\{v>0\}, \\ v(\cdot, 0)=0 . & \end{cases}
$$

Here $\delta$ is the Dirac $\delta$-function with mass at the origin, and the constant $C$ depends on $K$ and $n$. The constant $\langle 1 / g\rangle$ represents the "average" of $1 / g$ and it will be properly defined later.

The following heuristics can help with understanding of the free boundary velocity for the limit problem. For the moment, we suppose that $g$ is periodic and the solution $v$ is sufficiently smooth. 
Since the free boundary $\partial\{v>0\}$ is a level set of $v$, its normal velocity is given by $V=v_{t} /|D v|$. If we interpret (HS) as a quasi-stationary limit of the one-phase Stefan problem where $v$ is the temperature and $-D v$ is the heat flux, the quantity $1 / g$ represents the latent heat of the phase transition depending on the position (see [21]). Indeed, for the free boundary to expand by $\mathrm{d} x$, the heat flux must deliver $(1 / g) \mathrm{d} x$ of heat into the interface. Since it delivers exactly $|D v| \mathrm{d} t$ of heat in time $\mathrm{d} t$, we have

$$
V \sim \frac{\mathrm{d} x}{\mathrm{~d} t}=\frac{1}{1 / g(x)}|D v|=g(x)|D v| .
$$

When we rescale the problem, the latent heat will simply average out and therefore

$$
V=\frac{1}{\langle 1 / g\rangle}|D v|
$$

is the natural free boundary velocity of the limit solution.

A result similar to ours was obtained in [23] for weak solutions of the homogeneous Hele-Shaw problem with the free boundary velocity

$$
V=|D v| .
$$

In the current situation, however, the velocity law of the free boundary depends on the position and therefore the techniques from [23] can only provide lower and upper bounds on the free boundary radius. This requires the use of a more refined method to prove the convergence of the solution to the self-similar asymptotic profile. We combine the strengths of two notions of solution of (HS)viscosity and weak - using their correspondence studied in [14].

We use the weak solution $u(x, t): \mathbb{R}^{n} \times[0, \infty) \rightarrow \mathbb{R}$ considered in [9] by applying the transform

$$
u(x, t)=\int_{0}^{t} v(x, s) \mathrm{d} s .
$$

This transform was introduced for the porous dam problem in [3] (see also [4]) and for the onephase Stefan problem in [8]. For more details on the variational inequality framework, see also the survey [26]. The function $u$ formally solves the Euler-Lagrange equation

$$
\begin{cases}-\Delta u=-\frac{1}{g(x, \omega)} \chi_{\mathbb{R}^{n} \backslash \Omega_{0}} & \text { in }\{u>0\}, \\ u=|D u|=0 & \text { on } \partial\{u>0\}, \\ u=t & \text { on } K\end{cases}
$$

of some obstacle problem. Since $g$ satisfies (1.1) and (1.2), the subadditive ergodic theorem (see $[1,7])$ implies that there is a constant, denoted $\langle 1 / g\rangle$, such that

$$
\int_{\mathbb{R}^{n}} \frac{1}{g\left(\lambda^{1 / n} x\right)} u(x) \mathrm{d} x \rightarrow \int_{\mathbb{R}^{n}}\left\langle\frac{1}{g}\right) u(x) \mathrm{d} x \quad \text { for a.e. } \omega \in A
$$

as $\lambda \rightarrow \infty$. This then leads to the homogenization of the obstacle problem (1.5).

While in [14] the uniform convergence of the weak solutions in the homogenization limit follows more directly from the regularity of the obstacle problem (1.5), the current situation is considerably different. As the fixed domain $K$ shrinks to the origin due to the rescaling, the rescaled weak solution 
gets singular. Thus the convergence of weak solutions as $\lambda \rightarrow \infty$ is restricted to a locally uniform convergence on compact sets not containing the origin and the obstacle problem regularity cannot be used directly. We resolve this difficulty by introducing a limit obstacle problem and studying its wellposedness. The main challenge is posed by the singular behavior of the solution as $|x| \rightarrow 0$. From this development, we can deduce the necessary locally uniform convergence away from the origin. As the last step, we extend the applicability of the tools developed in [14] to our situation with a singularity at the origin. This allows us to conclude that the rescaled free boundary converges uniformly to the free boundary of the asymptotic profile, a sphere.

The current method requires $g(x, \omega)$ to be strictly positive, the main reason being the loss of uniqueness of viscosity solutions if $g$ becomes zero and the free boundary can stop. It is an interesting open problem if $g$ can reach zero yet $1 / g \in L_{\text {loc }}^{1}$ so that (1.6) still makes sense. It is currently under investigation by the author. The interpretation (1.4) of (HS) in terms of heat balance suggests that the homogenized velocity law $V=\langle 1 / g\rangle^{-1}|D v|$ continues to hold, at least for maximal viscosity solutions.

As noted above, the Hele-Shaw problem can be thought of as a quasi-stationary limit of the one-phase Stefan problem. The results obtained in $[18,23]$ on the asymptotic behavior of the (homogeneous) one-phase Stefan problem show that a solution of the Stefan problem converges to a solution of the (homogeneous) Hele-Shaw problem as $t \rightarrow \infty$. Furthermore, the homogenization result for an inhomogeneous one-phase Stefan problem was proved in [15]. This suggests an interesting question: does a solution of such a Stefan problem have a rescaling and an asymptotic behavior as $t \rightarrow \infty$ which is similar to our result for (HS)?

A brief outline follows: Section 2 summarizes the two notions of solution for problem (HS) and their correspondence. In Section 3, we introduce the rescaling of (HS), which is then followed by an investigation of radially symmetric test functions in Section 4 . The main contribution of the paper is contained in Sections 5 and 6, where we first define and study the limit obstacle problem and then show that the rescaled weak solutions of (HS) converge to the unique solution of the limit obstacle problem. Finally, equipped with those results in Section 7, we turn back to the viscosity notion to prove the uniform convergence of free boundaries to those of the limit problem.

\section{Hele-Shaw type problem}

Notation. Since the arguments are independent of $\omega$, we fix $\omega \in A$ for which all (1.1), (1.2) and (1.6) hold and we omit $\omega$ in the rest of the paper.

Throughout the article, we will make use of the standard notation for the bilinear form $a_{\Omega}(\cdot, \cdot)$ on $H^{1}(\Omega)$ and the scalar product $\langle\cdot, \cdot\rangle_{\Omega}$ on $L^{2}(\Omega)$ for some domain $\Omega$,

$$
a_{\Omega}(u, v)=\int_{\Omega} D u \cdot D v \mathrm{~d} x, \quad\langle u, v\rangle_{\Omega}=\int_{\Omega} u v \mathrm{~d} x .
$$

Whenever $\Omega=\mathbb{R}^{n}$, we drop the subscript $\Omega$.

For a set $A$, we denote by $A^{c}$ its complement. Given a nonnegative function $v$, we respectively define the positivity set of $v$ and the free boundary of $v$,

$$
\Omega(v):=\{(x, t): v(x, t)>0\}, \quad \Gamma(v):=\partial \Omega(v),
$$

and their time-slices

$$
\Omega_{t}(v):=\{x: v(x, t)>0\}, \quad \Gamma_{t}(v):=\partial \Omega_{t}(v) .
$$


$(f)_{+}$will be the positive part of $f$, i.e.

$$
(f)_{+}=\max \{f, 0\} .
$$

Lastly, $B_{r}(x)$ is the open space ball of radius $r$ centered at $x$,

$$
B_{r}(x)=\left\{y \in \mathbb{R}^{n}:|y-x|<r\right\} .
$$

This section reviews the two notions of solution of (HS) that will be used in this paper.

The notion of viscosity solution for (HS) was introduced in [13] and used in [14] to prove the uniform convergence of the free boundary in the homogenization limit. We will consider solutions on the spacetime cylinder $Q=\left(\mathbb{R}^{n} \backslash K\right) \times[0, \infty)$.

DEFINITION 2.1 A nonnegative upper-semicontinuous function $v$ defined in $Q$ is a viscosity subsolution of (HS) if

(a) for each $T>0$, the set $\overline{\Omega(v)} \cap\{t \leqslant T\} \cap Q$ is bounded, and

(b) for every $\phi \in C_{x, t}^{2,1}(Q)$ such that $v-\phi$ has a local maximum in $\overline{\Omega(v)} \cap\left\{t \leqslant t_{0}\right\} \cap Q$ at $\left(x_{0}, t_{0}\right)$, the following two conditions hold:

(i) if $v\left(x_{0}, t_{0}\right)>0$ then $-\Delta \phi\left(x_{0}, t_{0}\right) \leqslant 0$,

(ii) if $\left(x_{0}, t_{0}\right) \in \Gamma(v),|D \phi|\left(x_{0}, t_{0}\right) \neq 0$ and $-\Delta \phi\left(x_{0}, t_{0}\right)>0$, then

$$
\left(\phi_{t}-g\left(x_{0}\right)|D \phi|^{2}\right)\left(x_{0}, t_{0}\right) \leqslant 0 .
$$

DEFINITION 2.2 A nonnegative lower-semicontinuous function $v$ defined in $Q$ is a viscosity supersolution of (HS) if for every $\phi \in C_{x, t}^{2,1}(Q)$ such that $v-\phi$ has a local minimum in $\overline{\Omega(v)} \cap\left\{t \leqslant t_{0}\right\} \cap Q$ at $\left(x_{0}, t_{0}\right)$, the following two conditions hold:

(a) if $v\left(x_{0}, t_{0}\right)>0$ then $-\Delta \phi\left(x_{0}, t_{0}\right) \geqslant 0$,

(b) if $\left(x_{0}, t_{0}\right) \in \Gamma(v),|D \phi|\left(x_{0}, t_{0}\right) \neq 0$ and $-\Delta \phi\left(x_{0}, t_{0}\right)<0$, then

$$
\left(\phi_{t}-g\left(x_{0}\right)|D \phi|^{2}\right)\left(x_{0}, t_{0}\right) \geqslant 0 .
$$

Now we can define viscosity sub- and supersolutions with the appropriate initial and boundary data.

DEFINITION 2.3 A viscosity subsolution $v$ of (HS) in $Q$ is a viscosity subsolution of (HS) in $Q$ with initial data $v_{0}$ and boundary data 1 if

(a) $v$ is upper-semicontinuous in $\bar{Q}, v=v_{0}$ at $t=0$ and $v \leqslant 1$ on $\Gamma$,

(b) $\overline{\Omega(v)} \cap\{t=0\}=\overline{\left\{x: v_{0}(x)>0\right\}}$.

DEFINITION 2.4 A viscosity supersolution $v$ of (HS) in $Q$ is a viscosity supersolution of (HS) in $Q$ with initial data $v_{0}$ and boundary data 1 if $v$ is lower-semicontinuous in $\bar{Q}, v=v_{0}$ at $t=0$ and $v \geqslant 1$ on $\Gamma$.

Finally, we can define a viscosity solution:

DEFinition 2.5 A viscosity supersolution $v$ of (HS) in $Q$ (with initial data $v_{0}$ and boundary data 1 ) is a viscosity solution of (HS) in $Q$ (with initial data $v_{0}$ and boundary data 1 ) if

$$
v^{\star}(x, t):=\limsup _{(y, s) \rightarrow(x, t)} v(y, s)
$$

is a viscosity subsolution of (HS) in $Q$ (with initial data $v_{0}$ and boundary data 1 ). 
The following comparison principle is given in [13]:

THEOREM 2.6 ( $\left[13\right.$, Theorem 1.7]) Let $v_{1}$ and $v_{2}$ be respectively a viscosity subsolution and a viscosity supersolution of (HS). If $\overline{\left\{v_{1}(\cdot, 0)>0\right\}}$ is bounded, $v_{1}(x, 0)<v_{2}(x, 0)$ in $\left\{v_{1}(\cdot, 0)>0\right\}$ and $v_{1}<v_{2}$ on $\partial K \times[0, \infty)$, then

$$
v_{1} \leqslant v_{2} \quad \text { on } K^{c} \times[0, \infty) .
$$

The second notion of solution is the one introduced by [9], given by an obstacle problem for each time for a new variable $u(x, t)=\int_{0}^{t} v(x, s) \mathrm{d} s$ :

DEFINITION 2.7 The function $u(x, t)$ is called a weak solution of problem (HS) if for every $t \geqslant 0$, $w=u(\cdot, t)$ solves the obstacle problem

$$
\left\{\begin{array}{l}
w \in \mathcal{K}_{t}, \\
a(w, \varphi-w) \geqslant\left\langle-\frac{1}{g(x)} \chi_{\mathbb{R}^{n} \backslash \Omega_{0}}, \varphi-w\right\rangle \text { for all } \varphi \in \mathcal{K}_{t},
\end{array}\right.
$$

where

$$
\mathcal{K}_{t}=\left\{\varphi \in H_{0}^{1}\left(\mathbb{R}^{n}\right): \varphi \geqslant 0 \text { in } \Omega, \varphi=t \text { on } K\right\} .
$$

The theory of the obstacle problem (2.1) is well understood. We refer the reader to [25] for instance. We have the following:

Proposition 2.8 Suppose that $K \subset \Omega_{0} \subset \mathbb{R}^{n}, \Omega_{0}$ is a bounded open set and $K$ is a compact set with $\partial K \in C^{1,1}$. Then (2.1) has a unique solution $u(\cdot, t) \in H_{0}^{1}\left(\mathbb{R}^{n}\right)$ for all $t>0$. For every $T>0$ there exists $R=R(T)$ such that

$$
\Omega_{t}(u) \subset B_{R}(0) \quad \text { for all } t \in[0, T] .
$$

Moreover, for any $1<p<\infty$, there is a constant $C=C(m, n, p)$ such that

$$
\|u(\cdot, t)\|_{W^{2, p}\left(\mathbb{R}^{n} \backslash K\right)} \leqslant C t \quad \text { for all } t \geqslant 0 .
$$

Finally, the function $t \mapsto u(x, t)$ is Lipschitz continuous with

$$
\left|u\left(x, t_{1}\right)-u\left(x, t_{2}\right)\right| \leqslant\left|t_{1}-t_{2}\right| \text { for all } 0<t_{1} \leqslant t_{2}, x \in \mathbb{R}^{n} .
$$

One of the main tools used in this paper is the following correspondence of weak and viscosity solutions proved in [14]:

THEOREM 2.9 (cf. [14, Theorem 3.1]) Let $u(x, t)$ be the unique solution of (2.1). For each $t>0$, let $v(\cdot, t)$ be the solution of

$$
\begin{cases}\Delta v(\cdot, t)=0 & \text { in } \Omega_{t}(u) \backslash K, \\ v=1 & \text { on } K, \\ v=0 & \text { on } \Gamma_{t}(u),\end{cases}
$$

i.e. for every $t>0, v(\cdot, t)$ is the supremum of all lower-semicontinuous functions $w(x)$ for which there exists some $s, 0<s<t$, such that

$$
\begin{cases}-\Delta w \leqslant 0 & \text { in } \Omega_{s}(u), \\ w=1 & \text { on } K, \\ \operatorname{supp} w \subset \Omega_{S}(u) . & \end{cases}
$$


Then $v$ is a viscosity solution of (HS). Moreover,

$$
v(x, t)=\partial_{t}^{-} u(x, t),
$$

the left time derivative of $u$.

\section{Rescaling}

We are interested in the behavior of (HS) as $t \rightarrow \infty$. The goal is to prove that a rescaled solution converges to the self-similar solution of the homogeneous Hele-Shaw problem with a point delta source. As was observed in [23], the behavior depends on the dimension and the necessary rescaling is more involved in the two-dimensional case.

\subsection{Case $n \geqslant 3$}

The heat interpretation (1.4) of the free boundary velocity from the introduction suggests that the radius of the free boundary should behave as $\sim t^{1 / n}$ and thus we use the rescaling

$$
v^{\lambda}(x, t)=\lambda^{(n-2) / n} v\left(\lambda^{1 / n} x, \lambda t\right) .
$$

If we define $K^{\lambda}:=K / \lambda^{1 / n}$ and $\Omega_{0}^{\lambda}:=\Omega_{0} / \lambda^{1 / n}$, we derive the following problem for $v^{\lambda}$ from (HS):

$$
\begin{cases}-\Delta v^{\lambda}=0 & \text { in } \Omega\left(v^{\lambda}\right) \backslash K^{\lambda}, \\ v^{\lambda}=\lambda^{(n-2) / n} & \text { on } K^{\lambda}, \\ v_{t}^{\lambda}=g^{\lambda}(x)\left|D v^{\lambda}\right|^{2} & \text { on } \Gamma\left(v^{\lambda}\right), \\ v^{\lambda}(\cdot, 0)=v_{0}^{\lambda} & \text { on } \Omega_{0}^{\lambda} \backslash K^{\lambda},\end{cases}
$$

where $g^{\lambda}(x)=g\left(\lambda^{1 / n} x\right)$ as can be easily seen from

$$
\begin{aligned}
\lambda^{(n-2) / n+1} v_{t}\left(\lambda^{1 / n} x, \lambda t\right) & =g^{\lambda}(x) \lambda^{2(n-2) / n+2 / n}\left|(D v)\left(\lambda^{1 / n} x, \lambda t\right)\right|, \\
v_{t}\left(\lambda^{1 / n} x, \lambda t\right) & =g^{\lambda}(x)\left|(D v)\left(\lambda^{1 / n} x, \lambda t\right)\right|
\end{aligned}
$$

compared to the boundary condition in (HS)

$$
v_{t}\left(\lambda^{1 / n} x, \lambda t\right)=g\left(\lambda^{1 / n} x\right)\left|(D v)\left(\lambda^{1 / n} x, \lambda t\right)\right| .
$$

The rescaled problem (3.2) implies the weak formulation for the rescaled $u^{\lambda}(\cdot, t)=$ $\int_{0}^{t} v^{\lambda}(\cdot, s) \mathrm{d} s$, the obstacle problem

$$
\left\{\begin{array}{l}
w \in \mathcal{K}_{t}^{\lambda}, \\
a(w, \varphi-w) \geqslant\left\langle-\left(1 / g^{\lambda}\right) \chi_{\mathbb{R}^{n} \backslash \Omega_{0}^{\lambda}}, \varphi-w\right\rangle \quad \text { for all } \varphi \in \mathcal{K}_{t}^{\lambda},
\end{array}\right.
$$

for each $t>0$, where

$$
\mathcal{K}_{t}^{\lambda}=\left\{\varphi \in H^{1}\left(\mathbb{R}^{n}\right): \varphi \geqslant 0, \varphi=\lambda^{(n-2) / n} t \text { on } K^{\lambda}\right\} .
$$


The relation of $u^{\lambda}$ and $u$ can be deduced from the definition of $u^{\lambda}$ as

$$
\begin{aligned}
u^{\lambda}(x, t) & =\int_{0}^{t} v^{\lambda}(x, s) \mathrm{d} s=\lambda^{(n-2) / n} \int_{0}^{t} v\left(\lambda^{1 / n} x, \lambda s\right) \mathrm{d} s \\
& =\lambda^{(n-2) / n} \int_{0}^{\lambda t} v\left(\lambda^{1 / n} x, \sigma\right) \frac{\mathrm{d} \sigma}{\lambda}=\lambda^{-2 / n} u\left(\lambda^{1 / n} x, \lambda t\right) .
\end{aligned}
$$

The following observation will be useful:

LEMMA 3.1 The harmonic function $p(x)=C|x|^{2-n}$ is invariant under the rescaling (3.1). In particular, if $v(x, t) \leqslant C|x|^{2-n}$ for all $t>0$ then also

$$
v^{\lambda}(x, t) \leqslant C|x|^{2-n} \text { for all } t>0 \text {. }
$$

Proof. Observe that

$$
p^{\lambda}(x)=C \lambda^{(n-2) / n}\left|\lambda^{1 / n} x\right|^{2-n}=C|x|^{2-n}
$$

\subsection{Case $n=2$}

Since the rescaling (3.1) does not recover the logarithmic singularity at the origin in dimension $n=2$, we use a different rescaling that preserves the system:

$$
v^{\lambda}(x, t)=\log \mathcal{R}(\lambda) v(\mathcal{R}(\lambda) x, \lambda t),
$$

where $\mathcal{R}(\lambda)$ is the unique solution of

$$
\mathcal{R}^{2} \log \mathcal{R}=\lambda
$$

for any $\lambda>0$. In fact, the solution can be found explicitly in terms of the Lambert $\mathrm{W}$ function, $\mathcal{R}(\lambda)=\exp \left(\frac{1}{2} W(2 \lambda)\right)$. It is easy to see that $\lim _{\lambda \rightarrow \infty} \mathcal{R}(\lambda)=\infty$. Taking the logarithm of (3.6) gives

$$
2 \log \mathcal{R}(\lambda)\left(1+\frac{\log \log \mathcal{R}(\lambda)}{2 \log \mathcal{R}(\lambda)}-\frac{\log \lambda}{2 \log \mathcal{R}(\lambda)}\right)=0 .
$$

We deduce

$$
\lim _{\lambda \rightarrow \infty} \frac{\log \lambda}{2 \log \mathcal{R}(\lambda)}=1
$$

which together with (3.6) yields

$$
\lim _{\lambda \rightarrow \infty} \frac{\mathcal{R}(\lambda)}{\mathcal{R}_{\infty}(\lambda)}=1, \quad \mathcal{R}_{\infty}(\lambda)=\left(\frac{2 \lambda}{\log \lambda}\right)^{1 / 2} .
$$

In this case, we define $K^{\lambda}:=K / \mathcal{R}(\lambda)$ and $\Omega_{0}^{\lambda}:=\Omega_{0} / \mathcal{R}(\lambda)$. Since $v$ satisfies (HS), $v^{\lambda}$ satisfies

$$
\begin{cases}-\Delta v^{\lambda}=0 & \text { in } \Omega\left(v^{\lambda}\right) \backslash K^{\lambda}, \\ v^{\lambda}=\log \mathcal{R}(\lambda) & \text { on } K^{\lambda}, \\ v_{t}^{\lambda}=g^{\lambda}(x)\left|D v^{\lambda}\right|^{2} & \text { on } \Gamma\left(v^{\lambda}\right), \\ v^{\lambda}(\cdot, 0)=v_{0}^{\lambda} & \text { on } \Omega_{0}^{\lambda} \backslash K^{\lambda}\end{cases}
$$

Here $g^{\lambda}(x)=g(\mathcal{R}(\lambda) x)$, which can be derived as in (3.3) together with (3.6). 
The rescaled problem (3.9) implies a weak formulation for the rescaled $u^{\lambda}(x, t)=\int_{0}^{t} v^{\lambda}(x, s) \mathrm{d} s$ of the form (3.4) where now

$$
\mathcal{K}_{t}^{\lambda}=\left\{\varphi \in H^{1}\left(\mathbb{R}^{n}\right): \varphi \geqslant 0, \varphi=\log \mathcal{R}(\lambda) t \text { on } K^{\lambda}\right\} .
$$

This induces scaling for the weak solution $u$ analogous to (3.5),

$$
u^{\lambda}(x, t)=\frac{\log \mathcal{R}(\lambda)}{\lambda} u(\mathcal{R}(\lambda) x, \lambda t) .
$$

REMARK 3.2 Note that the scaling in dimensions $n \geqslant 3$ and the scaling in dimension $n=2$ are qualitatively different. Indeed, in dimension $n \geqslant 3$ a solution with a point source and a solution with a finite source have the same asymptotic speed of the free boundary $\approx t^{1 / n}$. That is not true in dimension $n=2$ where a point source solution has asymptotic speed $\approx t^{1 / 2}$, but a solution with a finite source has a slower asymptotic speed $\approx(t / \log t)^{1 / 2}$.

\section{Comparison with radially symmetric solutions}

The radially symmetric solutions for the Hele-Shaw problem, derived in [23], will serve as test functions in our arguments. A radially symmetric solution in the domain $|x| \geqslant a, t \geqslant 0$ is a pair of functions $p(x, t)$ and $R(t)$, where $p$ is of the form

$$
p(x, t)=\frac{A a^{2-n}\left(|x|^{2-n}-R^{2-n}(t)\right)_{+}}{a^{2-n}-R^{2-n}(t)} \text { if } n \geqslant 3,
$$

or

$$
p(x, t)=\frac{A\left(\log \frac{R(t)}{|x|}\right)_{+}}{\log \frac{R(t)}{a}} \quad \text { if } n=2,
$$

and $R(t)$ satisfies a certain algebraic equation (see [23] for details; we will be interested only in the behavior as $t \rightarrow \infty$ ). This solution satisfies the boundary conditions

$$
\begin{array}{rlrl}
p(x, t) & =A a^{2-n} & & \text { for }|x|=a>0, \\
p(x, t) & =0 & & \text { for }|x|=R(t), \\
R^{\prime}(t) & =\frac{1}{L}|D p| & & \text { for }|x|=R(t), \\
R(0) & =b>a . &
\end{array}
$$

Also

or

$$
\lim _{t \rightarrow \infty} \frac{R(t)}{c_{\infty} t^{1 / n}}=1, \quad c_{\infty}=\left(\frac{A n(n-2)}{L}\right)^{1 / n} \quad \text { if } n \geqslant 3,
$$

$$
\lim _{t \rightarrow \infty} \frac{R(t)}{c_{\infty}(t / \log t)^{1 / 2}}=1, \quad c_{\infty}=2 \sqrt{A / L} \quad \text { if } n=2 .
$$

In dimension $n=2$, we will also make use of the limit behavior

$$
\lim _{t \rightarrow \infty} \frac{\log R(t)}{\log t}=\frac{1}{2}
$$


LEMMA 4.1 For $L=1 / m$ (resp. $L=1 / M)$, with $m, M$ defined in $(1.1), p(x, t)$ is a viscosity subsolution (resp. supersolution) of (HS) in $Q$.

Proof. Assume that $1 / L=m$. The case $1 / L=M$ is similar. We will show that $p(x, t)$ satisfies the conditions in Definition 2.1.

$p(x, t)$ is clearly continuous and (a) immediately follows from (4.1a) and (4.3).

As for (b), (i) follows from the fact that $p(\cdot, t)$ is harmonic in $\Omega_{t}(p)$ for every $t \geqslant 0$.

To show (ii), let $\phi$ be a smooth function such that $p-\phi$ has a local maximum 0 in $\overline{\Omega(p)} \cap\left\{t \leqslant t_{0}\right\}$ at $\left(x_{0}, t_{0}\right) \in \Gamma(p)$ such that $|D \phi|\left(x_{0}, t_{0}\right) \neq 0$ and $-\Delta \phi\left(x_{0}, t_{0}\right)>0$. Let $B_{r} \subset \Omega_{t_{0}}(p)$ be a ball touching $\Gamma_{t_{0}}(p)$ from the inside at $\left(x_{0}, t_{0}\right)$ in which $-\Delta \phi>0$ (by smoothness of boundaries and continuity of $\Delta \phi$ there is such a ball). Then by Hopf's Lemma (see [10, Ch. 6.4.2]), since $-\Delta(\phi-p)=-\Delta \phi<0$,

$$
\frac{\partial(\phi-p)}{\partial v}>0 \quad \text { at }\left(x_{0}, t_{0}\right)
$$

where $v$ is the inner normal. Thus together with the fact that the boundaries are smooth level sets, we conclude that

$$
|D p|\left(x_{0}, t_{0}\right)=\frac{\partial p}{\partial v}<\frac{\partial \phi}{\partial v}=|D \phi|\left(x_{0}, t_{0}\right) .
$$

The normal velocity of the free boundary is given as $V_{n}=p_{t} /|D p|$ (level sets), and we have

$$
\frac{p_{t}}{|D p|}\left(x_{0}, t_{0}\right) \geqslant \frac{\phi_{t}}{|D \phi|}\left(x_{0}, t_{0}\right)
$$

Hence finally

$$
\begin{aligned}
\left(\phi_{t}-g\left(x_{0}\right)|D \phi|^{2}\right)\left(x_{0}, t_{0}\right) & <|D \phi|\left(\frac{p_{t}}{|D p|}-g\left(x_{0}\right)|D p|\right)\left(x_{0}, t_{0}\right) \\
& \stackrel{(1.1)}{\leqslant}|D \phi|\left(\frac{p_{t}}{|D p|}-m|D p|\right)\left(x_{0}, t_{0}\right) \stackrel{(4.2)}{=} 0 .
\end{aligned}
$$

Comparison with the radially symmetric sub- and supersolution provides us with the following straightforward result:

LEMMA 4.2 Let $v$ be a viscosity solution of (HS). There exists $t_{0}>0$ and constants $\rho_{1}$ and $\rho_{2}$, $0<\rho_{1}<\rho_{2}$, such that

$$
\rho_{1} t^{1 / n}<\min _{\Gamma_{t}(v)}|x| \leqslant \max _{\Gamma_{t}(v)}|x|<\rho_{2} t^{1 / n} \quad \text { if } n \geqslant 3,
$$

or

$$
\rho_{1} \mathcal{R}(t)<\min _{\Gamma_{t}(v)}|x| \leqslant \max _{\Gamma_{t}(v)}|x|<\rho_{2} \mathcal{R}(t) \quad \text { if } n=2,
$$

for $t \geqslant t_{0}$, and

$$
\max _{\Gamma_{t}(v)}|x|<\rho_{2}
$$

for $0 \leqslant t \leqslant t_{0}$. Moreover there is a constant $C>0$ such that

$$
0 \leqslant v(x, t) \leqslant C|x|^{2-n} .
$$




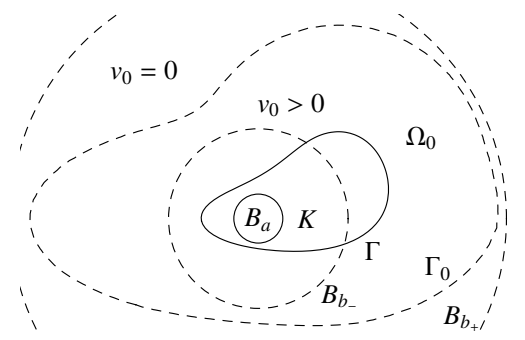

FIG. 2. Arrangement of domains for comparison.

Proof. Given problem (HS), we can find constants $a, b_{ \pm}$such that $B_{a}(0) \subset K$ and $\overline{B_{b_{-}}(0)} \subset$ $\Omega_{0} \subset \overline{\Omega_{0}} \subset B_{b_{+}}(0)$ (see Fig. 2). Set also $L_{-}=1 / m, L_{+}=1 / M$. Using the maximum principle for harmonic functions, we can also find constants $A_{-}$and $A_{+}$so that $p_{-}$and $p_{+}$, the radially symmetric subsolution and supersolution, respectively, given in (4.1) that satisfy the boundary conditions (4.2) with the respective constants, also satisfy

$$
p_{-} \prec v \prec p_{+} \quad \text { on }\left(K^{c} \times\{0\}\right) \cup(\Gamma \times(0, \infty)) \text {. }
$$

Then by the comparison principle for viscosity solutions [14, Theorem 2.6], we have

$$
p_{-} \prec v \prec p_{+} \quad \text { on } K^{c} \times(0, \infty)
$$

and also

$$
v(x, t) \leqslant p_{+}(x, t)<C|x|^{2-n}
$$

for $C>0$ large enough and

$$
R_{-}(t)<\min _{\Gamma_{t}(v)}|x| \leqslant \max _{\Gamma_{t}(v)}|x|<R_{+}(t),
$$

where $R_{ \pm}$are the radii of the free boundaries of the functions $p_{ \pm}$, respectively, and their limit behavior is given by (4.3).

Lemma 4.2 is all we need to prove the crucial theorem from [23]:

THEOREM 4.3 (Near-field limit) The viscosity solution $v(x, t)$ of the Hele-Shaw like problem (HS) converges to the unique solution $P(x)$ of the exterior Dirichlet problem

$$
\begin{cases}\Delta P=0, & x \in \mathbb{R}^{n} \backslash K, \\ P=1, & x \in \Gamma, \\ \lim _{|x| \rightarrow \infty} P(x)=0 & \text { if } n \geqslant 3, \text { or } \\ P \text { is bounded } & \text { if } n=2,\end{cases}
$$

as $t \rightarrow \infty$ uniformly on compact subsets of $\overline{K^{c}}$.

Proof. See proof of [23, Theorem 4.1].

The following constant $C_{*}$ characterizes the singularity of the limit solution: 
LEMmA 4.4 There exists a constant $C_{*}=C_{*}(K)$ such that the solution $P$ of problem (4.5) satisfies

$$
\lim _{|x| \rightarrow \infty}|x|^{n-2} P(x)=C_{*} .
$$

Proof. See Lemma 4.5 in [23].

Finally, we also need to improve on the convergence result for radially symmetric solutions from [23]:

LEMMA 4.5 Let $p(x, t)$ be a radially symmetric solution (4.1) of the Hele-Shaw problem satisfying the boundary conditions (4.2) with constants $A, a, b$ and $L$. Then the rescaled solutions $p^{\lambda}(x, t)$ converge locally uniformly on the set $\left(\mathbb{R}^{n} \backslash\{0\}\right) \times[0, \infty)$ to the solution of the Hele-Shaw problem with a point source,

$$
V(x, t)=V_{A, L}(x, t)= \begin{cases}A\left(|x|^{2-n}-\rho^{2-n}(t)\right)_{+}, & n \geqslant 3 \\ A\left(\log \frac{\rho(t)}{|x|}\right)_{+}, & n=2\end{cases}
$$

where

$$
\rho(t)=\rho_{L}(t)= \begin{cases}(A n(n-2) t / L)^{1 / n}, & n \geqslant 3, \\ (2 A t / L)^{1 / 2}, & n=2 .\end{cases}
$$

Proof. We shall show the uniform convergence on the sets $\{(x, t):|x| \geqslant \varepsilon, 0 \leqslant t \leqslant T\}$ for some $\varepsilon, T>0$. Let $t_{0}=\rho^{-1}(\varepsilon / 2)$. Starting with $n \geqslant 3$, we have

$$
p^{\lambda}(x, t)=\frac{A a^{2-n}\left(|x|^{2-n}-\left(\frac{R(\lambda t)}{(\lambda t)^{1 / n}} t^{1 / n}\right)^{2-n}\right)_{+}}{a^{2-n}-R^{2-n}(\lambda t)} .
$$

There are two cases:

(a) $t_{0} \leqslant t \leqslant T$. Due to (4.3), we have

$$
\frac{R(\lambda t)}{\lambda^{1 / n}}=\frac{R(\lambda t)}{(\lambda t)^{1 / n}} t^{1 / n} \rightarrow\left(\frac{A a^{n-2} n(n-2) t}{L}\right)^{1 / n}=\rho(t), \quad R^{2-n}(\lambda t) \rightarrow 0,
$$

as $\lambda \rightarrow \infty$ uniformly on $t_{0} \leqslant t \leqslant T$. That shows the uniform convergence $p^{\lambda} \rightarrow V$ on $\left\{|x| \geqslant \varepsilon, t_{0} \leqslant t \leqslant T\right\}$.

(b) $0 \leqslant t \leqslant t_{0}$. Clearly $V(x, t)=0$ in $\left\{|x| \geqslant \varepsilon, 0 \leqslant t \leqslant t_{0}\right\}$. Since

$$
\frac{R(\lambda t)}{\lambda^{1 / n}} \leqslant \frac{R\left(\lambda t_{0}\right)}{\lambda^{1 / n}}<\rho\left(t_{0}\right)+\frac{\varepsilon}{2}=\varepsilon
$$

for all $\lambda$ large enough, we see that $p^{\lambda}=0=V$ on $\left\{|x| \geqslant \varepsilon, 0 \leqslant t \leqslant t_{0}\right\}$ for all $\lambda$ large.

In dimension $n=2$, the rescaling yields

$$
p^{\lambda}(x, t)=A \frac{\left(\log \frac{R(\lambda t)}{\mathcal{R}(\lambda)|x|}\right)+}{\frac{\log (R(\lambda t) / a)}{\log \mathcal{R}(\lambda)}}
$$


Again, we split the proof into two cases:

(a) $t_{0} \leqslant t \leqslant T$. Rewriting $R(\lambda t) / \mathcal{R}(\lambda)$ and using (4.3b) and (3.8), we obtain

$$
\frac{R(\lambda t)}{\mathcal{R}(\lambda)}=\frac{R(\lambda t)}{\left(\frac{\lambda t}{\log \lambda t}\right)^{1 / 2}} \cdot \frac{\left(\frac{\lambda t}{\log \lambda t}\right)^{1 / 2}}{\left(\frac{\lambda}{\log \lambda}\right)^{1 / 2}} \cdot \frac{\left(\frac{\lambda}{\log \lambda}\right)^{1 / 2}}{\mathcal{R}(\lambda)} \rightarrow 2\left(\frac{A}{L}\right)^{1 / 2} \cdot t^{1 / 2} \cdot \frac{1}{\sqrt{2}}=\left(\frac{2 A t}{L}\right)^{1 / 2}
$$

as $\lambda \rightarrow \infty$ uniformly for $t_{0} \leqslant t \leqslant T$. Similarly, (4.4) and (3.7) lead to

$$
\frac{\log \frac{R(\lambda t)}{a}}{\log \mathcal{R}(\lambda)}=\frac{\log R(\lambda t)-\log a}{\log \lambda t} \cdot \frac{\log \lambda t}{\log \lambda} \cdot \frac{\log \lambda}{\log \mathcal{R}(\lambda)} \rightarrow \frac{1}{2} \cdot 1 \cdot 2=1
$$

as $\lambda \rightarrow \infty$ uniformly for $t_{0} \leqslant t \leqslant T$. This proves the uniform convergence $p^{\lambda} \rightarrow V$ on $\left\{|x| \geqslant \varepsilon, t_{0} \leqslant t \leqslant T\right\}$.

(b) $0 \leqslant t \leqslant t_{0}$. Argue as in the case $n \geqslant 3$, (b).

\section{The limit problem}

Our current task is a characterization of the limit of rescaled weak solutions $u^{\lambda}$ as $\lambda \rightarrow \infty$. We want to show that the limit satisfies a certain obstacle problem that can be interpreted as a Hele-Shaw problem with a point source. Existence and uniqueness of such a problem in two dimensions was studied in [6]. Our situation requires extending the definition to all dimensions $n \geqslant 3$.

First define $U_{A, L}(x, t)$ to be the Baiocchi transform of $V_{A, L}(x, t)$, introduced in Lemma 4.5 (see proof of Theorem 5.1 for derivation):

$$
U_{A, L}(x, t)== \begin{cases}\left(A t|x|^{2-n}+\frac{L}{2 n}|x|^{2}-\frac{1}{2}(A n t)^{2 / n}\left(\frac{n-2}{L}\right)^{(2-n) / n}\right)_{+} & \text {if } n \geqslant 3, \\ \left(\frac{A}{2} t \log \frac{2 A t}{L e|x|^{2}}+\frac{L|x|^{2}}{4}\right)_{+} & \text {if } n=2 .\end{cases}
$$

We say that $U(x, t)$ is a solution of the limit problem if for every $t \in[0, T], U(\cdot, t)$ satisfies the following obstacle problem:

$$
\begin{cases}w \in \mathcal{K}_{t}, & \\ a(w, \phi) \geqslant\langle-L, \phi\rangle, & \forall \phi \in V, \\ a(w, \psi w)=\langle-L, \psi w\rangle, & \forall \psi \in W,\end{cases}
$$

where

$$
\begin{aligned}
\mathcal{K}_{t} & =\left\{\varphi \in \bigcap_{\varepsilon>0} H^{1}\left(\mathbb{R}^{n} \backslash B_{\varepsilon}\right) \cap C\left(\mathbb{R}^{n} \backslash B_{\varepsilon}\right): \varphi \geqslant 0, \lim _{|x| \rightarrow 0} \frac{\varphi(x)}{U_{A, L}(x, t)}=1\right\}, \\
V & =\left\{\phi \in H^{1}\left(\mathbb{R}^{n}\right): \phi \geqslant 0, \phi=0 \text { on } B_{\varepsilon}(0) \text { for some } \varepsilon>0\right\}, \\
W & =V \cap C^{1}\left(\mathbb{R}^{n}\right) .
\end{aligned}
$$

TheOREM 5.1 For given $A, L>0, U_{A, L}(x, t)$ defined in (5.1) is the unique solution of the problem (5.2). 
Proof. We first verify that $U_{A, L}$ is a solution. It can be found by integrating the function $V_{A, L}(x, t)$ from Lemma 4.5 ,

$$
U(x, t)=\int_{0}^{t} V(x, s) \mathrm{d} s= \begin{cases}0 & t \leqslant s(x), \\ \int_{s(x)}^{t} V(x, t) \mathrm{d} t, & t>s(x),\end{cases}
$$

where

$$
s(x)=\rho^{-1}(|x|) .
$$

It is clear that $\Delta U=L$ in $U>0$ and $U=\partial U / \partial v=0$ on $\Gamma_{t}(U)$, i.e. when $|x|=\rho(t)$. A straightforward application of Green's theorem then shows that $U$ satisfies the variational equality and inequality in (5.2).

To prove the uniqueness, we will use a comparison for an obstacle problem and for this we need the following observation:

LEMMA 5.2 If $w$ satisfies the obstacle problem (5.2), it also satisfies the obstacle problem

$$
a_{\Omega}(w, \varphi-w) \geqslant\langle-L, \varphi-w\rangle_{\Omega} \quad \text { for all } \varphi \in \mathcal{K},
$$

on $\Omega=\mathbb{R}^{n} \backslash B_{a}$ for every $a>0$, where

$$
\mathcal{K}=\left\{\varphi \in H^{1}(\Omega): \varphi \geqslant 0, \varphi=w \text { on } \partial B_{a}\right\} .
$$

Proof. Fix $\varphi \in \mathcal{K}$. Pick $0<\varepsilon<a$. Find $\psi \in C^{1}\left(\mathbb{R}^{n}\right), 0 \leqslant \psi \leqslant 1$, such that $\psi=0$ on $B_{a-\varepsilon}(0)$ and $\psi=1$ on $B_{a}(0)^{c}$. Then $\psi \in W$. Define

$$
\tilde{\varphi}= \begin{cases}\varphi & \text { on } B_{a}(0)^{c} \\ w & \text { otherwise }\end{cases}
$$

Since $\left.\varphi\right|_{\partial B_{a}(0)}=\left.w\right|_{\partial B_{a}(0)}$, we have $\tilde{\varphi} \in H^{1}\left(\mathbb{R}^{n} \backslash B_{a-\varepsilon}\right)$. Define

$$
\phi=(\psi-1) w+\tilde{\varphi} \in H^{1}\left(\mathbb{R}^{n}\right) .
$$

Clearly $\phi \geqslant 0$ and $\phi=0$ for $|x| \leqslant a-\varepsilon$. Therefore $\phi \in V$. From problem (5.2) we have

$$
a_{\Omega}(w, \varphi-w)=a(w, \phi-\psi w) \geqslant\langle-L, \phi-\psi w\rangle=\langle-L, \varphi-w\rangle_{\Omega} .
$$

This holds for every $\varphi \in \mathcal{K}$.

The main tool is the following lemma:

LEMMA 5.3 (Comparison for the limit problem) Let $w_{1}, w_{2}$ be two solutions of the obstacle problem (5.2) for some $t>0$ with $A_{1}, L_{1}$ resp. $A_{2}, L_{2}$. If $0<A_{1}<A_{2}$ and $L_{1} \geqslant L_{2}>0$ then

$$
w_{1} \leqslant w_{2} \quad \text { for all } x \neq 0 .
$$

Proof. Let $\varepsilon=\left(A_{2}-A_{1}\right) / 3>0$. If $n \geqslant 3$, there exists $a>0$ such that

$$
\left|\frac{w_{1}(x)}{|x|^{2-n}}-A_{1} t\right|<\varepsilon \quad \text { and } \quad\left|\frac{w_{2}(x)}{|x|^{2-n}}-A_{2} t\right|<\varepsilon \quad \text { for all }|x| \leqslant a .
$$


We can replace $|x|^{2-n}$ by $-\frac{1}{2} \log |x|$ in dimension $n=2$. In particular, $w_{1}(x) \leqslant w_{2}(x)$ in $|x| \leqslant a$. Lemma 5.2 implies that $w_{1}$ and $w_{2}$ satisfy the obstacle problem on $\Omega=B_{a}(0)^{c}$,

$$
a\left(w_{i}, \varphi-w_{i}\right)_{\Omega} \geqslant\left\langle-L_{i}, \varphi-w_{i}\right\rangle_{\Omega} \quad \forall \varphi \in \mathcal{K}_{i}, i=1,2,
$$

where $\mathcal{K}_{i}=\left\{\varphi \in H^{1}(\Omega): \varphi \geqslant 0, \varphi=w_{i}\right.$ on $\left.|x|=a\right\}$. Now we can use the comparison for the obstacle problem (see Corollary 5.2, Chapter 4 in [25]) to obtain $w_{1} \leqslant w_{2}$ in $\Omega$.

Now we can finish the proof of Theorem 5.1. Lemma 5.3 implies that for any $\varepsilon>0$ we can compare

$$
U_{A-\varepsilon, L}(x, t) \leqslant U(x, t) \leqslant U_{A+\varepsilon, L}(x, t),
$$

since $U_{A, L}(x, t)$ are solutions of the limit problem (5.2). After taking the limit $\varepsilon \rightarrow 0$ we conclude that

$$
U(x, t)=U_{A, L}(x, t) .
$$

\section{Uniform convergence of $u^{\lambda}$}

The following lemma was proven in [14]:

Lemma 6.1 (cf. [14, Lemma 4.1]) For given $g$ satisfying (1.1) and (1.2), there exists a constant, denoted $\langle 1 / g\rangle$, such that if $\Omega \subset \mathbb{R}^{n}$ is a bounded measurable set and if $\left\{u^{\varepsilon}\right\}_{\varepsilon>0} \subset L^{2}(\Omega)$ is a collection of functions such that $u^{\varepsilon} \rightarrow u$ strongly in $L^{2}(\Omega)$ as $\varepsilon \rightarrow 0$, then

$$
\lim _{\varepsilon \rightarrow 0} \int_{\Omega} \frac{1}{g(x / \varepsilon, \omega)} u^{\varepsilon}(x) \mathrm{d} x=\int_{\Omega}\left(\frac{1}{g}\right) u(x) \mathrm{d} x \quad \text { a.e. } \omega .
$$

Recall that in Section 2, we fixed $\omega$ for which (6.1) holds. The goal of this section is proving the following theorem:

THEOREM 6.2 The functions $u^{\lambda}$ converge to $U_{A, L}$ as $\lambda \rightarrow \infty$ locally uniformly on $\left(\mathbb{R}^{n} \backslash\{0\}\right) \times$ $[0, \infty)$, where $U_{A, L}$ is the unique solution of the limit problem from Theorem 5.1, with $A=C_{*}$ from Lemma 4.4, and $L=\langle 1 / g\rangle$ from Lemma 6.1.

Proof. We need to prove uniform convergence on the sets $\{|x| \geqslant \varepsilon, 0 \leqslant t \leqslant T\}$. Hence fix $T>0$ and $\varepsilon>0$. The functions $u^{\lambda}(\cdot, t)$ satisfy the obstacle problem (3.4) for each $t \geqslant 0$ and $\lambda>0$.

Using Lemma 4.2, we can find $\rho_{2}>0$ and $t_{0}>0$ such that

$$
\begin{array}{ll}
\Omega_{t}(u) \subset B_{\rho_{2}} \mathcal{R}(t) & \text { for all } t \geqslant t_{0}, \\
\Omega_{t}(u) \subset B_{\rho_{2}} & \text { for all } 0 \leqslant t \leqslant t_{0} .
\end{array}
$$

Inclusion (6.2) is preserved under rescaling when $n \geqslant 3$. In dimension $n=2$, we have

$$
\Omega_{t}\left(u^{\lambda}\right) \subset B_{\rho_{2}} \mathcal{R}(\lambda t) / \mathcal{R}(\lambda) \quad \text { for all } t \geqslant t_{0} / \lambda .
$$

But

$$
\frac{\mathcal{R}(\lambda t)}{\mathcal{R}(\lambda)} \leqslant \frac{\mathcal{R}(\lambda T)}{\mathcal{R}(\lambda)} \sim\left(\frac{T \log \lambda}{\log \lambda T}\right)^{1 / 2} \rightarrow T^{1 / 2} \quad \text { as } \lambda \rightarrow \infty .
$$

Thus we can find $\hat{\rho}>0$ such that $\Omega_{t}\left(u^{\lambda}\right) \subset \Omega:=B_{\hat{\rho}}(0)$ for all $0 \leqslant t \leqslant T$ and $\lambda>1$. Define $\Omega_{\varepsilon}:=\{x \in \Omega:|x| \geqslant \varepsilon\}$ and $Q_{\varepsilon}:=\Omega_{\varepsilon} \times[0, T]$. 
Now find $\lambda_{0}>1$ such that $K^{\lambda} \subset B_{\varepsilon / 2}(0)$ for all $\lambda \geqslant \lambda_{0}$. Since $u^{\lambda}(\cdot, t)$ is a solution of the rescaled obstacle problem (3.4), we can use the regularity estimates for the obstacle problem from [25, Theorem 5.2.4] to conclude that

$$
\left\|\Delta u^{\lambda}(\cdot, t)\right\|_{L^{q}\left(\Omega_{\varepsilon / 2}\right)} \leqslant\left\|\frac{1}{g^{\lambda}}\right\|_{L^{q}\left(\Omega_{\varepsilon / 2}\right)} \quad \text { for all } 1 \leqslant q \leqslant \infty .
$$

Let $p_{+}$be the radially symmetric supersolution from the proof of Lemma 4.2. Since by Lemma 4.5 the rescaled $p_{+}^{\lambda}$ converges uniformly on the set $Q_{\varepsilon / 2}$ to the function $V_{A, L}$ as $\lambda \rightarrow \infty$ for some $A$ and $L$, we can find a constant $C_{1}$ such that $p_{+}^{\lambda} \leqslant C_{1}$ on $Q_{\varepsilon / 2}$ for all $\lambda \geqslant \lambda_{0}$. Now recall that due to Theorem 2.9 we can express $u^{\lambda}$ as

$$
u^{\lambda}(x, t)=\int_{0}^{t} v^{\lambda}(x, s) \mathrm{d} s \leqslant \int_{0}^{t} p_{+}^{\lambda}(x, s) \mathrm{d} s \leqslant C_{1} T \quad \text { for }(x, t) \in Q_{\varepsilon / 2} .
$$

In particular, $\left\|u^{\lambda}(\cdot, t)\right\|_{L^{2}\left(\Omega_{\varepsilon / 2}\right)}$ is bounded uniformly in $t \in[0, T]$ and $\lambda \geqslant \lambda_{0}$.

Thus we can use the standard elliptic regularity results (see [16], for instance) to find constants $0<\alpha<1$ and $C_{2}$, independent of $t \in[0, T]$ and $\lambda \geqslant \lambda_{0}$, such that

$$
\begin{aligned}
\left\|u^{\lambda}(\cdot, t)\right\|_{H^{2}\left(\Omega_{\varepsilon}\right)} & \leqslant C_{2} \\
\left\|u^{\lambda}(\cdot, t)\right\|_{C^{0, \alpha}\left(\Omega_{\varepsilon}\right)} & \leqslant C_{2}
\end{aligned} \quad \text { for all } 0 \leqslant t \leqslant T, \lambda \geqslant \lambda_{0} .
$$

Using (6.3) again, we have $\left|u^{\lambda}(x, t)-u^{\lambda}(x, s)\right| \leqslant C_{3}|t-s|$ and we conclude that

$$
\left\|u^{\lambda}\right\|_{C^{0, \alpha}\left(Q_{\varepsilon}\right)} \leqslant C_{4}\left(C_{2}, C_{3}\right) \text { for all } \lambda \geqslant \lambda_{0} .
$$

Using the standard diagonalization argument and Arzelà-Ascoli, we can find a subsequence $u^{\lambda_{k}}$ that converges locally uniformly on $Q_{\varepsilon}, \varepsilon>0$, to a function $\bar{u}$ as $k \rightarrow \infty$. Due to the compact embedding of $H^{1}$ in $H^{2}$, the uniqueness of the limit and the bound on the $H^{2}$-norm implies that also $u^{\lambda_{k}}(\cdot, t) \rightarrow \bar{u}(\cdot, t)$ in $H^{1}$-norm on $Q_{\varepsilon}$ as $\lambda \rightarrow \infty$ for every $0 \leqslant t \leqslant T, \varepsilon>0$.

Finally, in the following two lemmas, we will show that $\bar{u}$ is a solution of the limit problem (5.2). Using the uniqueness of the limit problem (Theorem 5.1), we conclude that the convergence is not restricted to a subsequence and we have

$$
u^{\lambda} \rightarrow U_{C_{*},\langle 1 / g\rangle}, \quad \lambda \rightarrow \infty,
$$

locally uniformly on $\left(\mathbb{R}^{n} \backslash\{0\}\right) \times[0, \infty)$, which concludes the proof of Theorem 6.2.

LEMMA $6.3 \bar{w}=\bar{u}(\cdot, t)$ satisfies

$$
\begin{aligned}
a(\bar{w}, \phi) & \geqslant(-L, \phi), & \forall \phi \in V, \\
a(\bar{w}, \psi \bar{u}) & =(-L, \psi \bar{w}), & \forall \psi \in W,
\end{aligned}
$$

for each $0 \leqslant t \leqslant T$, where $L=\langle 1 / g\rangle$ is defined in Lemma 6.1 and $V$, $W$ were defined in (5.3a).

Proof. Fix $t \in[0, T]$ and $\phi \in V$. We will denote $w^{k} \equiv u^{\lambda_{k}}(x, t)$. There is $\varepsilon>0$ such that $\phi=0$ in $B_{\varepsilon}(0)$. There is also $k_{0}$ such that if $k \geqslant k_{0}$, we have $\Omega_{0}^{\lambda_{k}} \subset B_{\varepsilon}(0)$. Define $\varphi^{k}=\phi+w^{k}$. Clearly $\phi=0$ on $\Gamma_{0}^{\lambda_{k}}$ if $k \geqslant k_{0}$ and thus $\varphi^{k} \in \mathcal{K}_{t}^{\lambda_{k}}$. Since $u^{\lambda_{k}}$ satisfies (3.4), we have

$$
a\left(w^{k}, \phi\right)=a\left(w^{k}, \varphi^{k}-w^{k}\right) \geqslant\left\langle-\frac{1}{g^{\lambda_{k}}}, \varphi^{k}-w^{k}\right\rangle=\left\langle-\frac{1}{g^{\lambda_{k}}}, \phi\right\rangle .
$$


The mapping $w \mapsto a(w, \phi)$ is a linear functional on $H^{1}$ and hence $a\left(w^{k}, \phi\right) \rightarrow a(\bar{w}, \phi)$ as $k \rightarrow \infty$ because $w^{k} \rightarrow \bar{w} H^{1}$-strongly. Lemma 6.1 also yields $\left\langle-1 / g^{\lambda_{k}}, \phi\right\rangle \rightarrow\langle-L, \phi\rangle$. Hence we conclude

$$
a(\bar{w}, \phi) \geqslant\langle-L, \phi\rangle .
$$

Now fix $\psi \in W$ such that $0 \leqslant \psi \leqslant 1$. There is $B_{\varepsilon}(0)$ in which $\psi=0$ and $k_{0}$ as above. Define $\varphi_{k}=(1-\psi) w^{k}$. Again $\varphi_{k} \in \mathcal{K}_{t}^{\lambda_{k}}$ if $k \geqslant k_{0}$. Now

$$
a\left(w^{k}, \psi w^{k}\right)=-a\left(w^{k}, \varphi^{k}-w^{k}\right) \leqslant-\left\langle-\frac{1}{g^{\lambda_{k}}}, \varphi^{k}-w^{k}\right\rangle=\left\langle-\frac{1}{g^{\lambda_{k}}}, \psi w^{k}\right\rangle .
$$

The fact that $w^{k} \rightarrow \bar{w} L^{2}$-strongly implies $\psi w^{k} \rightarrow \psi \bar{w}$ in $L^{2}$, and by Lemma 6.1,

$$
\left\langle-\frac{1}{g^{\lambda_{k}}}, \psi w^{k}\right\rangle \rightarrow\langle-L, \psi \bar{w}\rangle
$$

Due to the lower semicontinuity of the map $w \mapsto a(w, \psi w)$ in $H^{1}$, we also obtain

$$
a(\bar{w}, \psi \bar{w}) \leqslant \liminf _{k \rightarrow \infty} a\left(w^{k}, \psi w^{k}\right) \leqslant \lim _{k \rightarrow \infty}\left\langle-\frac{1}{g^{\lambda_{k}}}, \psi w^{k}\right\rangle=\langle-L, \psi \bar{u}\rangle .
$$

Finally, since $\psi \bar{w} \in V$, we have

$$
a(\bar{w}, \psi \bar{w}) \leqslant\langle-L, \psi \bar{w}\rangle \leqslant a(\bar{w}, \psi \bar{w}) .
$$

LEMMA 6.4 We have

$$
\lim _{|x| \rightarrow 0} \frac{\bar{u}(x, t)}{U_{C_{*}, L}(x, t)}=1
$$

for every $t \geqslant 0$, where $C_{*}$ is the constant from Lemma 4.4.

Proof. We will use a comparison with the sub- and supersolutions from Lemma 4.1.

Let $C_{*}$ be the constant from Lemma 4.4. Fix $\varepsilon>0$. Then by Lemma 4.4 there exists $a>0$ laege enough such that

$$
\left|\frac{P(x)}{a^{2-n}}-C_{*}\right|<\frac{\varepsilon}{2} \quad \text { for }|x|=a .
$$

The set $\{|x|=a\}$ is a compact subset of $\mathbb{R}^{n} \backslash K$ and by the near-field limit (Theorem 4.3), there is $t_{0}>0$ large enough that

$$
\left|\frac{v(x, t)}{a^{2-n}}-\frac{P(x)}{a^{2-n}}\right|<\frac{\varepsilon}{2} \quad \text { for }|x|=a, t \geqslant t_{0} .
$$

Therefore we have

$$
\left|\frac{v(x, t)}{a^{2-n}}-C_{*}\right|<\varepsilon \quad \text { for }|x|=a, t \geqslant t_{0} .
$$

Let $p_{+}$and $p_{-}$be the radially symmetric supersolution, resp. subsolution, satisfying the boundary conditions

$$
\begin{aligned}
\frac{p_{ \pm}}{a^{2-n}} & =C_{*} \pm \varepsilon \quad \text { on }|x|=a \\
b_{+}=\max _{x \in \Gamma_{t_{0}}(v)}|x|, \quad b_{-} & =\min _{x \in \Gamma_{t_{0}}(v)}|x|, \quad L_{+}=\frac{1}{M}, \quad L_{-}=\frac{1}{m} .
\end{aligned}
$$


Then by a comparison,

$$
p_{-}\left(x, t-t_{0}\right) \leqslant v(x, t) \leqslant p_{+}\left(x, t-t_{0}\right) \quad \text { for }|x| \geqslant a, t \geqslant t_{0} .
$$

Lemma 4.5 yields

$$
p_{ \pm}^{\lambda} \rightarrow V_{ \pm}:=V_{C_{*} \pm \varepsilon, L_{ \pm}} \quad \text { as } \lambda \rightarrow \infty
$$

locally uniformly on $\{|x|>0, t \geqslant 0\}$.

Using the formula (3.5) for $u^{\lambda}$ we have, for $\lambda^{1 / n}|x| \geqslant|a|$ and $\lambda \geqslant t_{0} / t$,

$$
\begin{aligned}
u^{\lambda}(x, t) & =\int_{0}^{t} v^{\lambda}(x, s) \mathrm{d} s \\
& \leqslant \int_{0}^{t_{0} / \lambda} v^{\lambda}(x, s) \mathrm{d} s+\int_{t_{0} / \lambda}^{t} p_{+}^{\lambda}\left(x, s-t_{0} / \lambda\right) \mathrm{d} s \\
& =\int_{0}^{t_{0} / \lambda} v^{\lambda}(x, s) \mathrm{d} s+\int_{0}^{t-t_{0} / \lambda} p_{+}^{\lambda}(x, s) \mathrm{d} s
\end{aligned}
$$

The first term tends to 0 as $\lambda \rightarrow \infty$ since $v^{\lambda}(x, s) \leqslant C|x|^{2-n}$ by Lemma 4.2. The locally uniform convergence of $p_{+}^{\lambda}$ then implies that the second integral converges to $\int_{0}^{t} V_{+}(x, s) \mathrm{d} s$ as $\lambda \rightarrow \infty$ by the bounded convergence theorem. The same argument can be used to bound $u^{\lambda}$ from below using $p_{-}$and we finally have

$$
\begin{aligned}
\int_{0}^{t} V_{-}(x, s) \mathrm{d} s & \leqslant \liminf _{\lambda \rightarrow \infty} u^{\lambda}(x, t) \\
& \leqslant \bar{u}(x, t) \leqslant \limsup _{\lambda \rightarrow \infty} u^{\lambda}(x, t) \leqslant \int_{0}^{t} V_{+}(x, s) \mathrm{d} s
\end{aligned}
$$

for all $(x, t) \in\left(\mathbb{R}^{n} \backslash\{0\}\right) \times[0, \infty)$.

Now suppose that $n \geqslant 3$. The integrals on both sides can be found explicitly as in the proof of Theorem 5.1 and

$$
\int_{0}^{t} V_{ \pm}(x, s) \mathrm{d} s=U_{ \pm}(x, t):=U_{C_{*} \pm \varepsilon, L_{ \pm}}(x, t)
$$

with

$$
\lim _{|x| \rightarrow 0} \frac{U_{C_{*} \pm \varepsilon, L_{ \pm}}(x, t)}{|x|^{2-n}}=\left(C_{*} \pm \varepsilon\right) t .
$$

Then we divide (6.4) by $|x|^{2-n}$ and take the limit $|x| \rightarrow 0$, which leads to

$$
\left(C_{*}-\varepsilon\right) t \leqslant \liminf _{|x| \rightarrow 0} \frac{\bar{u}(x)}{|x|^{2-n}} \leqslant \limsup _{|x| \rightarrow 0} \frac{\bar{u}(x)}{|x|^{2-n}} \leqslant\left(C_{*}+\varepsilon\right) t .
$$

We obtain an analogous result in dimension $n=2$, after replacing $|x|^{2-n}$ by $-\log |x|$. The proof is complete because $\varepsilon>0$ was arbitrary.

This finishes the proof of Theorem 6.2. 


\section{Convergence of $v$ and the free boundary}

In this final section, we want to show the locally uniform convergence of $v^{\lambda}$ and $\Gamma\left(v^{\lambda}\right)$ as $\lambda \rightarrow \infty$. Define the half-relaxed limits

$$
v^{*}(x, t)=\limsup _{(y, s), \lambda \rightarrow(x, t), \infty} v^{\lambda}(y, s), \quad v_{*}(x, t)=\liminf _{(y, s), \lambda \rightarrow(x, t), \infty} v^{\lambda}(y, s),
$$

in $\{|x| \neq 0, t \geqslant 0\}$. Let $V(x, t)=V_{C_{*}, L}(x, t)$ be the radially symmetric solution with a point source at 0 from Lemma 4.5, where $C_{*}$ is the constant from Lemma 4.4 and $L=\langle 1 / g\rangle$. Clearly

$$
\Omega_{t}(V)=\{x: 0<|x|<\rho(t)\} .
$$

By continuity, $V_{*}=V=V^{*}$.

Our goal is proving a theorem similar to [14, Theorem 4.4].

THEOREM 7.1 $\left\{\Gamma\left(v^{\lambda}\right)\right\}_{\lambda}$ converges to $\Gamma(V)$ locally uniformly with respect to the Hausdorff distance, and $v^{\lambda}$ converges locally uniformly to $V$ in $\left(\mathbb{R}^{n} \backslash\{0\}\right) \times[0, \infty)$ as $\lambda \rightarrow \infty$, and furthermore

$$
v_{*}=v^{*}=V .
$$

We will use the ideas developed in [14]. The proof is somewhat less technical because $V$, the limit of $v^{\lambda}$, is smooth.

First, we will collect some necessary technical results in the spirit of [14]:

REMARK 7.2 (a) Since $v$ is a viscosity supersolution, $v^{\lambda}(\cdot, t)$ is superharmonic in $\Omega_{t}\left(v^{\lambda}\right)$ and therefore also $v_{*}(\cdot, t)$ is superharmonic in $\Omega_{t}\left(v_{*}\right)$.

(b) $u(\cdot, t)$ is a weak solution of $-\Delta w \leqslant 0$ for every $t>0$ and thus $v(x, t) \geqslant(1 / t) u(x, t)$.

(c) The construction of the viscosity solution $v$ implies that $v^{\lambda}$ is subharmonic in $\left(K^{\lambda}\right)^{c}$ (see [14, proof of Theorem 3.1, Step 2]). Therefore $v^{*}$ is subharmonic in $\mathbb{R}^{n} \backslash\{0\}$.

Lemma 7.3 (cf. [14, Lemma 4.6]) Suppose $\left(x_{k}, t_{k}\right) \in\left\{u^{\lambda_{k}}=0\right\}$ and $\left(x_{k}, t_{k}, \lambda_{k}\right) \rightarrow\left(x_{\infty}, t_{\infty}, \infty\right)$ with $x_{0} \neq 0$. Then the following holds:

(a) $\left(x_{0}, t_{0}\right) \in\{U=0\}$.

(b) If $x_{k} \in \Gamma_{t_{k}}\left(u^{\lambda_{k}}\right)$ then $x_{\infty} \in \Gamma_{t_{\infty}}(U)$.

We need to identify the singularity of $v_{*}$ and $v^{*}$ at $x=0$.

LEMma $7.4 v^{*}$ and $v_{*}$ defined above have a singularity at 0 with

$$
\lim _{|x| \rightarrow 0+} \frac{v_{*}(x, t)}{V(x, t)}=1, \quad \lim _{|x| \rightarrow 0+} \frac{v^{*}(x, t)}{V(x, t)}=1
$$

for each $t>0$.

Proof. Fix $\varepsilon>0$. Using comparison with the radially symmetric subsolution $p_{-}$and supersolution $p_{+}$as in Lemma 6.4, with

$$
\frac{p_{ \pm}(x, t)}{a^{2-n}}=C_{*} \pm \varepsilon \quad \text { for }|x|=a,
$$

we deduce that there are $a>0$ and $t_{0}>0$ such

$$
p_{-}\left(x, t-t_{0}\right) \leqslant v(x, t) \leqslant p_{+}\left(x, t-t_{0}\right) \quad \text { for all } t \geqslant t_{0},|x| \geqslant a .
$$


Since

$$
p_{ \pm}^{\lambda}\left(x, t-t_{0} / \lambda\right) \rightarrow V_{C_{*} \pm \varepsilon, L_{ \pm}}(x, t) \quad \text { as } \lambda \rightarrow \infty,
$$

locally uniformly on the set $\left(\mathbb{R}^{n} \backslash\{0\}\right) \times[0, \infty)$, the uniform convergence yields

$$
V_{C_{*}-\varepsilon, L_{-}}(x, t) \leqslant v_{*}(x, t) \leqslant v^{*}(x, t) \leqslant V_{C_{*}+\varepsilon, L_{+}}(x, t) .
$$

We have the conclusion because $\varepsilon>0$ was arbitrary.

LEMMA 7.5 (cf. [14, Lemma 4.12]) There exists a constant $C_{1}=C_{1}(n, M)$ such that if $\left(x_{0}, t_{0}\right) \in$ $\Omega\left(v^{\lambda}\right)$ and $B_{r}\left(x_{0}\right) \cap \Omega_{0}^{\lambda}=\emptyset$, we have

$$
\sup _{B_{r}\left(x_{0}\right)} v^{\lambda}\left(\cdot, t_{0}\right) \geqslant \frac{C_{1} r^{2}}{t_{0}} .
$$

Proof. Recall that $v^{\lambda}\left(\cdot, t_{0}\right) \geqslant u^{\lambda}\left(\cdot, t_{0}\right) / t_{0}$ (Remark 7.2) and use Lemma 3.3 of [14].

LEMMA 7.6 (cf. [14, Lemma 4.10]) The following inclusion holds:

$$
\Omega(V) \subset \Omega\left(v_{*}\right) .
$$

Moreover,

$$
v_{*} \geqslant V
$$

Proof. Recall that $v(x, t) \geqslant(1 / t) u(x, t)$ for all $t>0, x \neq 0$ and that the inequality is preserved under rescaling (3.1) and (3.5). As $u^{\lambda}$ converges to $U$ uniformly on each set $\{|x|>\varepsilon\} \times[0, T]$, we have

$$
v_{*}(x, t) \geqslant \frac{1}{t} U(x, t)
$$

and we see that $\Omega(V)=\Omega(U) \subset \Omega\left(v_{*}\right)$.

Recall that $v_{*}(\cdot, t)$ is superharmonic in $\Omega_{t}\left(v_{*}\right)$ for each $t$ (Remark 7.2) and behaves at zero as $V(\cdot, t)$ by Lemma 7.4. For fixed $\varepsilon>0$, comparison of $v_{*}(\cdot, t)$ and $V_{C_{*}-\varepsilon, L}(\cdot, t)$ yields

$$
v_{*}(x, t) \geqslant V_{C_{*}-\varepsilon, L}(x, t) \quad \text { for every } t>0,
$$

because $\Omega\left(V_{C_{*}-\varepsilon, L}\right) \subset \Omega(V)$. We conclude by taking the limit $\varepsilon \rightarrow 0$.

LEMMA 7.7 (cf. [14, Lemma 4.13(ii)]) The following inclusion of boundaries holds:

$$
\Gamma\left(v^{*}\right) \subset \Gamma(V) .
$$

Proof. Argue as in [14], with the use of Lemma 7.3 above.

Proof of Theorem 7.1. Since $\Omega_{t}\left(v^{*}\right)$ is bounded for every time $t>0$ by Lemma 4.2, and $\Omega(V)$ is a simply connected set, Lemma 7.7 also implies that in fact $\Omega\left(v^{*}\right) \subset \overline{\Omega(V)}$. In particular, we see that

$$
\overline{\Omega\left(v^{*}\right)} \subset \overline{\Omega(V)} \subset \Omega\left(V_{C_{*}+\varepsilon, L}\right) \text { for all } \varepsilon>0 .
$$

Recall that $v^{*}(\cdot, t)$ is subharmonic in $\mathbb{R}^{n} \backslash\{0\}$ for every $t>0$ (Remark 7.2). Since $\lim _{|x| \rightarrow 0} v^{*}(x, t) / V(x, t)=1$ for all $t \geqslant 0$, we see that $v^{*}(x, t) \leqslant V_{C_{*}+\varepsilon, L}(x, t)$ for every $\varepsilon>0$. After letting $\varepsilon \rightarrow 0+$, we recover

$$
V(x, t) \leqslant v_{*}(x, t) \leqslant v^{*}(x, t) \leqslant V(x, t)
$$


and

$$
\Gamma\left(v_{*}\right)=\Gamma\left(v^{*}\right)=\Gamma(V) .
$$

To prove the locally uniform convergence of boundaries with respect to the Hausdorff distance, fix $0<t_{1}<t_{2}$ and denote

$$
\Gamma^{\lambda}=\Gamma\left(v^{\lambda}\right) \cap\left\{t_{1} \leqslant t \leqslant t_{2}\right\}, \quad \Gamma^{\infty}=\Gamma(V) \cap\left\{t_{1} \leqslant t \leqslant t_{2}\right\} .
$$

We define the $\delta$-neighborhood of a set $A \subset \mathbb{R}^{n} \times \mathbb{R}$ by

$$
U_{\delta}(A):=\{(x, t): \operatorname{dist}((x, t), A)<\delta\} .
$$

We need to show that for every $\delta>0$ there exists $\lambda_{0}>0$ such that

$$
\begin{array}{cc}
\Gamma^{\lambda} \subset U_{\delta}\left(\Gamma^{\infty}\right) & \forall \lambda \geqslant \lambda_{0}, \\
\Gamma^{\infty} \subset U_{\delta}\left(\Gamma^{\lambda}\right) & \forall \lambda \geqslant \lambda_{0} .
\end{array}
$$

First, suppose that for some $\delta>0$, the inclusion (7.1) fails infinitely often. In other words, suppose that there is $\delta>0$ and a sequence $\left\{\lambda_{k}\right\}_{k=1}^{\infty}$ with $\lambda_{k} \rightarrow \infty$ such that

$$
\Gamma^{\lambda_{k}} \cap\left(U_{\delta}\left(\Gamma^{\infty}\right)\right)^{c} \neq \emptyset .
$$

Accordingly, we choose a sequence of points $\left(x_{k}, t_{k}\right) \in \Gamma^{\lambda_{k}}$ whose distance from $\Gamma^{\infty}$ is bounded by $\delta$ from below,

$$
\operatorname{dist}\left(\left(x_{k}, t_{k}\right), \Gamma^{\infty}\right) \geqslant \delta
$$

Since $\bigcup_{\lambda} \Gamma^{\lambda}$ is bounded (recall Lemma 4.2), there is a converging subsequence $\left(x_{k_{j}}, t_{k_{j}}\right) \rightarrow\left(x_{0}, t_{0}\right)$ as $j \rightarrow \infty$. Moreover, $x_{0} \neq 0$ by Corollary 4.2 and also $t_{1} \leqslant t_{0} \leqslant t_{2}$. Hence Lemma 7.3(b) implies that $\left(x_{0}, t_{0}\right) \in \Gamma(U)=\Gamma(V)$ and therefore $\left(x_{0}, t_{0}\right) \in \Gamma^{\infty}$. But that is a contradiction with (7.3).

To prove the second inclusion, (7.2), we start by proving a pointwise result. Suppose that there is $\delta>0$, a point $\left(x_{0}, t_{0}\right) \in \Gamma^{\infty}$ and a sequence $\left\{\lambda_{k}\right\}_{k=1}^{\infty}$ with $\lambda_{k} \rightarrow \infty$ such that $\operatorname{dist}\left(\left(x_{0}, t_{0}\right), \Gamma^{\lambda_{k}}\right) \geqslant \delta / 2$ for every $k$. That means that there is $r>0$ such that the spacetime cylinder $D_{r}\left(x_{0}, t_{0}\right):=B_{r}\left(x_{0}\right) \times\left[t_{0}-r, t_{0}+r\right]$ satisfies either

$$
D_{r}\left(x_{0}, t_{0}\right) \subset\left\{v^{\lambda_{k}}>0\right\} \quad \text { or } \quad D_{r}\left(x_{0}, t_{0}\right) \subset\left\{v^{\lambda_{k}}=0\right\} .
$$

At least one of the inclusions holds for infinitely many $k$. Therefore we arrive at two possibilities:

(a) In the case of the first inclusion, Lemma 7.5 and Harnack's inequality in $B_{r / 2}\left(x_{0}\right)$ for all $t \in$ $\left[t_{0}-r, t_{0}+r\right]$ yield

$$
\frac{C_{1} r^{2}}{4 t} \leqslant \sup _{B_{r / 2}\left(x_{0}\right)} v^{\lambda_{k}}(\cdot, t) \leqslant C_{2} \inf _{B_{r / 2}\left(x_{0}\right)} v^{\lambda_{k}}(\cdot, t),
$$

where $C_{2}$ does not depend on $\lambda_{k}$ and $t$. We conclude that $v^{*}>0$ in $B_{r / 2}\left(x_{0}\right) \times\left[t_{0}-r, t_{0}+r\right]$ and that is a contradiction with $\left(x_{0}, t_{0}\right) \in \Gamma^{\infty} \subset \Gamma\left(v^{*}\right)$.

(b) In the second case, we clearly have $v_{*}=0$ in $D_{r}\left(x_{0}, t_{0}\right)$, which contradicts $\left(x_{0}, t_{0}\right) \in$ $\Gamma^{\infty} \subset \Gamma\left(v_{*}\right)$. 
We conclude that for each $\delta>0$ and $\left(x_{0}, t_{0}\right) \in \Gamma^{\infty}$ there is $\lambda_{0}$, depending on $\left(x_{0}, t_{0}\right)$, such that $\operatorname{dist}\left(\left(x_{0}, t_{0}\right), \Gamma^{\lambda}\right)<\delta / 2$ for all $\lambda \geqslant \lambda_{0}$. But if $(x, t) \in \Gamma^{\infty}$ and $\left|(x, t)-\left(x_{0}, t_{0}\right)\right|<\delta / 2$, we have

$$
\operatorname{dist}\left((x, t), \Gamma^{\lambda}\right) \leqslant \operatorname{dist}\left(\left(x_{0}, t_{0}\right), \Gamma^{\lambda}\right)+\left|(x, t)-\left(x_{0}, t_{0}\right)\right|<\delta \quad \forall \lambda \geqslant \lambda_{0} .
$$

The set $\Gamma^{\infty}$ is compact and therefore it can be covered by finitely many open sets,

$$
\Gamma^{\infty} \subset \bigcup_{1 \leqslant j \leqslant j_{0}}\left\{(x, t):\left|(x, t)-\left(x_{0}^{j}, t_{0}^{j}\right)\right|<\delta / 2\right\} .
$$

We can set $\lambda_{0}=\max _{1 \leqslant j \leqslant j_{0}} \lambda_{0}^{j}$ to conclude that

$$
\Gamma^{\infty} \subset U_{\delta}\left(\Gamma^{\lambda}\right) \quad \forall \lambda \geqslant \lambda_{0} .
$$

\section{Acknowledgments}

This work is part of my doctoral research. I would like to sincerely thank my thesis advisor, Inwon

C. Kim, for suggesting the problem and for her invaluable advice and support.

\section{REFERENCES}

1. Akcoglu, M. A., \& Krengel, U. Ergodic theorems for superadditive processes. J. Reine Angew. Math. 323 (1981), 53-67. Zbl 0453.60039 MR 0611442

2. Antontsev, S. N., Gonçalves, C. R., \& Meirmanov, A. M. Local existence of classical solutions to the well-posed Hele-Shaw problem. Portugal Math. (N.S.) 59 (2002), 435-452. Zbl 1037.35100 MR 1942849

3. BaiocChi, C. Su un problema di frontiera libera connesso a questioni di idraulica. Ann. Mat. Pura Appl. (4) 92 (1972), 107-127. Zbl 0258.76069 MR 0408443

4. BAIOCCHI, C., \& CAPElo, A. Variational and Quasivariational Inequalities. Wiley, New York (1984). Zbl 0551.49007 MR 0745619

5. Caffarelli, L. A., Souganidis, P. E., \& Wang, L. Homogenization of fully nonlinear, uniformly elliptic and parabolic partial differential equations in stationary ergodic media. Comm. Pure Appl. Math. 58 (2005), 319-361. Zbl 1063.35025 MR 2116617

6. Č́́ŽEK, P., \& JANOvsKÝ, V. Hele-Shaw flow model of the injection by a point source. Proc. Roy. Soc. Edinburgh Sect. A 91 (1981), 147-159. Zbl 0508.76030 MR 0648924

7. Dal Maso, G., \& ModicA, L. Nonlinear stochastic homogenization and ergodic theory. J. Reine Angew. Math. 368 (1986), 28-42. Zbl 0582.60034 MR 0850613

8. Duvaut, G. Résolution d'un problème de Stefan (fusion d'un bloc de glace à zéro degré). C. R. Acad. Sci. Paris Sér. A-B 276 (1973), A1461-A1463. Zbl 0258.35037 MR 0328346

9. Elliott, C. M., \& JanovskÝ, V. A variational inequality approach to Hele-Shaw flow with a moving boundary. Proc. Roy. Soc. Edinburgh Sect. A 88 (1981), 93-107. Zbl 0455.76043 MR 0611303

10. Evans, L. C. Partial Differential Equations. Grad. Stud. Math. 19, Amer. Math. Soc., Providence, RI (2010). Zbl 1194.35001 MR 2597943

11. Friedman, A., \& Kinderlehrer, D. A one phase Stefan problem. Indiana Univ. Math. J 24 (1975), 1005-1035. Zbl 0334.49002 MR 0385326

12. Hele-Shaw, H. S. The flow of water. Nature $\mathbf{5 8}$ (1897), 467-468. JFM 29.0646.01

13. KIM, I. C. Homogenization of the free boundary velocity. Arch. Ration. Mech. Anal. 185 (2007), 69-103. Zbl 1162.35011 MR 2308859 
14. Kim, I. C., \& Mellet, A. Homogenization of a Hele-Shaw type problem in periodic and random media. Arch. Ration. Mech. Anal. 194 (2009), 507-530. Zbl pre05640834 MR 2563637

15. Kim, I. C., \& Mellet, A. Homogenization of one-phase Stefan-type problems in periodic and random media. Trans. Amer. Math. Soc. 362 (2010), 4161-4190. Zbl 1197.35290 MR 2608400

16. Ladyzhenskaya, O. A., \& Ural'tseva, N. N. Linear and Quasilinear Elliptic Equations. Math. Sci. Engrg. 46, Academic Press, New York and London (1968). Zbl 0164.13002 MR 0244627

17. Louro, B., \& Rodrigues, J.-F. Remarks on the quasisteady one phase Stefan problem. Proc. Roy. Soc. Edinburgh Sect. A 102 (1986), 263-275. Zbl 0608.35081 MR 0852360

18. Matano, H. Asymptotic behavior of the free boundaries arising in one-phase Stefan problems in multidimensional spaces. In: Nonlinear Partial Differential Equations in Applied Science (Tokyo, 1982), North-Holland Math. Stud. 81, North-Holland, Amsterdam (1983), 133-151. Zbl 0564.35100 MR 0730240

19. McGeough, J. A., \& Rasmussen, H. On the derivation of the quasi-steady model in electrochemical machining. J. Inst. Math. Appl. 13 (1974), 13-21.

20. Papanicolaou, G. C., \& Varadhan, S. R. S. Boundary value problems with rapidly oscillating random coefficients. In: Random Fields, Vol. I, II (Esztergom, 1979), Colloq. Math. Soc. János Bolyai 27, North-Holland, Amsterdam (1981), 835-873. Z Zbl 0499.60059 MR 0712714

21. Primicerio, M. Stefan-like problems with space-dependent latent heat. Meccanica 5 (1970), 187-190. Zbl 0207.10001 MR 0372424

22. Primicerio, M., \& Rodrigues, J.-F. The Hele-Shaw problem with nonlocal injection condition. In: Nonlinear Mathematical Problems in Industry, II (Iwaki, 1992), GAKUTO Int. Ser. Math. Sci. Appl. 2, Gakkōtosho, Tokyo (1993), 375-390. Z Zbl 0875.35157 MR 1370478

23. Quirós, F., \& VÁzQuez, J. L. Asymptotic convergence of the Stefan problem to Hele-Shaw. Trans. Amer. Math. Soc. 353 (2001), 609-634. Zbl 0956.35136 MR 1804510

24. RichARDSON, S. Hele-Shaw flows with a free boundary produced by the injection of fluid into a narrow channel. J. Fluid Mech. 56 (1972), 609-618. Zbl 0256.76024

25. Rodrigues, J.-F. Obstacle Problems in Mathematical Physics. North-Holland Math. Stud. 134, Elsevier (1987). Zbl 0606.73017 MR 0880369

26. Rodrigues, J.-F. The variational inequality approach to the one-phase Stefan problem. Acta Appl. Math. 8 (1987), 1-35. Zbl 0653.35083 MR 0871691

27. RoubíčEk, T. The Stefan problem in heterogeneous media. Ann. Inst. H. Poincaré Anal. Non Linéaire 6 (1989), 481-501. Z Zbl 0706.35139 MR 1035339

28. Steinbach, J. A Variational Inequality Approach to Free Boundary Problems with Applications in Mould Filling, Int. Ser. Numer. Math. 136, Birkhäuser, Basel (2002). Z Zbl 1011.35001 MR 1891393 